\title{
Transfusion Medicine in Switzerland
}

\author{
Guy Levy ${ }^{\mathrm{a}}$ Christa Byland $^{\mathrm{b}} \quad$ Christoph Niederhauser $^{\mathrm{b}} \quad$ Rudolf Schwabe $^{\mathrm{a}} \quad$ Marianne Senn $^{\mathrm{c}}$ \\ Behrouz Mansouri Taleghani ${ }^{\mathrm{b}}$
}

${ }^{a}$ Executive Board of the Blood Transfusion Service SRC,

${ }^{\mathrm{b}}$ Regional Blood Transfusion Service Bern LTD,

c Swissmedic (Haemovigilance), Berne, Switzerland

\section{Key Words}

Laws · Guidelines · Haemovigilance - Immunological risks . Residual risk

\section{Summary}

Swiss transfusion medicine currently encompasses $13 \mathrm{Re}$ gional Blood Transfusion Services (RBTS) under the Blood Transfusion Service of the Swiss Red Cross Ltd (BTS SRC) which is responsible for quality standards, logistics, and reference activities in immunohaematology and testing for infectious diseases, as well as haematological divisions of several university hospitals. Switzerland covers currently its own needs of labile blood components. According to the law, blood components are drugs and must comply with the actual legal requirements. Furthermore, the BTS SRC has published mandatory guidelines for all RBTS to ensure the standardisation of used methods. Haemovigilance is in charge of Swissmedic and covers all aspects of transfusion medicine, from donor selection to blood transfusion. Measures for preventing immunological and non-immunological risks as well as transfusion-transmitted diseases are also implemented at all stages of the process. The last preventive measures are universal pre-storage leucocyte depletion (in 1999), exclusion from blood donation of individuals who stayed longer than 6 months in the UK (in 2002) or have previously been transfused (in 2004). Mandatory screening of blood donations includes anti-HIV 1/2, HIV NAT, anti-HCV, HCV NAT, HBsAg, anti-Treponema pallidum and alaninaminotransferase. In the last years the calculated residual risk for transfusion-transmitted HIV or HCV was approximately 1:2-3 million and for HBV approximately 1: 200,000-300,000. Future challenges are the maintenance of self-sufficiency, the sustained recruitment of dedicated staff and the rising costs.

\author{
Schlüsselwörter \\ Gesetze · Richtlinien · Hämovigilanz · Immunologische \\ Risiken · Restrisiko
}

\section{Zusammenfassung}

Die Transfusionsmedizin der Schweiz umfasst 13 Regionale Blutspendedienste (RBSD) unter dem Dach des Blutspendedienstes des Schweizerischen Roten Kreuzes (BSD SRK), der die Verantwortung für die Qualitätsstandards, Logistik und Referenztätigkeiten in der Immunhämatologie und bei den Infektmarkertests trägt, sowie die entsprechend spezialisierten Einheiten der Universitätsspitäler. Die Schweiz deckt aktuell ihren Eigenbedarf an labilen Blutpräparaten. Nach dem Gesetz gelten Blutpräparate als Arzneimittel und müssen die entsprechend geltenden Anforderungen erfüllen. Ferner hat der BSD SRK für die RBSD verpflichtende Vorschriften publiziert, um die Anwendung standardisierter Methoden zu gewährleisten. Die Swissmedic ist für die Hämovigilanz verantwortlich, die alle Aspekte der Transfusionsmedizin einschließt, von der Spenderauswahl bis zur Transfusion. Maßnahmen zur Verhütung von immunologischen und nichtimmunologischen Risiken sowie von transfusionsassoziierten Infektionen sind ebenfalls auf jeder Stufe des Gesamtprozesses implementiert. Die diesbezüglich zuletzt eingeführten Maßnahmen waren die generelle Leukozytendepletion vor der Lagerung (1999) sowie der Ausschluss von Personen von der Blutspende, die sich länger als 6 Monate in Großbritannien aufgehalten haben (2002) oder früher selbst transfundiert wurden (2004). Anlässlich jeder Blutspende müssen folgende Infektionsuntersuchungen durchgeführt werden: Anti-HIV 1/2, HIV-PCR, Anti-HCV, HCV-PCR, HBsAg, Anti-Treponema-pallidum-Antikörper und die Alaninaminotransferase. In den vergangenen Jahren lag das kalkulierte Restrisiko einer transfusionsbedingten Infektion mit HIV oder HCV bei etwa 1:2-3 Millionen sowie von HBV bei etwa 1:200 000-300 000 Transfusionen. Die zukünftigen Herausforderungen umfassen die Aufrechterhaltung der Selbstversorgung, die nachhaltige Rekrutierung von engagierten Mitarbeitern sowie die Kostenexpansion.

\begin{tabular}{ll}
\hline KARGER & $\oplus$ 2006 S. Karger GmbH, Freiburg \\
Fax +49 7614520714 & Accessible online at: \\
$\begin{array}{l}\text { E-mail Information@Karger.de } \\
\text { www.karger.com }\end{array}$ & www.karger.com/tmh \\
\end{tabular}

\section{KARGER}

www.karger.com
Prof. Dr. Guy Levy

Direction of Blood Transfusion Service SRC

Laupenstrasse 37, Postfach 5510, 3001 Bern, Switzerland

Tel +41 31380 81-81, Fax -80

E-mail guy.levy@redcross.ch 


\section{National Specifics}

\section{Introduction and History}

Switzerland is a typical federal country with about 7.5 million inhabitants, 3 main populations and languages (German 65\%, French $25 \%$ and Italian 10\%), federal institutions (Governmental College and Parliament) and 23 political independent districts ('cantons') keeping a large autonomy in decision making and monitoring their own health structures so far as it is allowed by the Fundamental Health Law.

In 1949, the Swiss Red Cross (SRC) set up a Central Laboratory (ZLB) in Berne for the preparation of blood products, and in 1951 the government mandated the SRC to supply the Swiss population with blood products in normal and war situation. Consequently, a majority of cantonal sections of the SRC decided to create blood transfusion centres (BTC) run by physicians of cantonal or local hospitals under the responsibility of the cantonal health authorities. As a result, each BTC developed its own culture depending on the local situation, with the ZLB keeping a leadership in the field of collection, preparation, research and development.

The situation changed dramatically in 1994, with a governmental requirement to put in place an organisation able to apply the same criteria about donor selection, quality and security of blood products and to manage adequately the blood supply all over the country. This goal was reached in several steps. Under the leadership of the SRC, mandatory guidelines were established, and 13 Regional Blood Transfusion Services (RBTS) were created: 5 facilities in the cities with a medical faculty and 8 others all over the rest of the country, performing the core activities. The other BTC were closed or, for a majority, limited their activities to blood collection. In 2000 , the ZLB, as a fractionation facility, was sold by the SRC to a foreign operator.

Today, the Blood Transfusion Service of the Swiss Red Cross Ltd (BTS SRC) is a not-for-profit organisation, collecting 370,000 blood donations per year in the above mentioned 13 RBTS whose donation volumes range between 12,000 and 100,000 blood donations per year. The BTS SRC has almost a monopolistic situation in the country, as only one small facility remains independent, run by a local hospital. Apheresis activities (mainly platelet collections) are performed both by RBTS and haematological divisions of several university hospitals. Switzerland currently covers its own needs and remains in a comfortable situation with a sufficient number of regular and new donors (but still a certain lack of young male donors). An increasing number of deferral reasons in the last few years lead to a diminution of the donor population which is remarkable but still not a threat.

The public perception of the BTS SRC and the 13 RBTS is excellent. Government, politicians and the public trust the organisation to maintain a safe and sufficient blood supply for the country.
The BTS SRC has a long lasting tradition of support programmes to countries or institutions in need of red cells. For many years it took part in the Euroblood program for the New York blood centre (the program was stopped in 2002), and since more than a decade red cells are sent to Athens, Greece, to support the local blood supply.

The standards of quality, logistics (national coordination system of planning and interregional exchange of blood components ensuring an adequate supply of all hospitals, even in case of bottle-neck situations during the holiday season) and reference activities in immunohaematology and serology (these last activities performed in a reference laboratory located in the facility of the RBTS Bern) are in charge of the BTS SRC.

\section{National Laws}

From 1951 until 1996, the only requirement of the federal authorities was summarised in a Federal Decree mentioning that the SRC was acknowledged as the only Red Cross society in the country and, as such, mandated to ensure the blood supply in peace and war time.

In 1996, the first federal regulation was published in order 'to warrant the security and all manipulations with blood and blood products for the protection of donors and recipients', implying the implementation of good manufacturing practice (GMP).

In 2002 this regulation was replaced by a federal Law on Therapeutic Products (LTP) applying to synthetic human drugs, biotechnology, vaccines, medical devices, implants, diagnostic agents and blood products. Special dispositions concern blood and blood components whose preparation must comply with the requirements in place for drugs. Furthermore, criteria for donor selection, mandatory tests, traceability and record keeping are defined. The regulations refer also to the guide of the Council of Europe [1] and to the PIC/S GMP guide [2].

The law set up a federal drug regulatory agency (Swissmedic) whose decisions are mandatory all over the country, which guarantees quality, safety and efficiency of drugs and performs regular inspections of all BTC preparing and releasing blood components. After each inspection the BTC receive an official licence in order to carry out their activity. The main points to be considered are:

- A designated person in charge to ensure that blood and blood components meet the specifications and that collection, preparation and distribution of blood and blood components comply with the regulations for GMP.

- The blood transfusion service must maintain a quality management system which fulfils the requirements of GMP.

- The blood transfusion service must nominate a person in charge of haemovigilance who notifies all adverse events to Swissmedic. 
As a result of these requirements, preparation of blood components today is obviously standardised, and the quality is ensured. But the appropriate use of these components is not regulated by law: national transfusion guidelines per se do not exist in Switzerland.

\section{Standards, Quality and Accreditation}

\section{National Guidelines / Standards / Quality Policies}

National guidelines (so-called 'Prescriptions of the BTS SRC') were published for the first time in 1996, at the same time as the first federal regulation. They provided to every BTC the regulatory basic elements of GMP dealing with both production and quality management requirements. In 2000, the board of RBTS directors and the executive board of the BTS SRC decided to adapt their own quality management system according to the international standards EN ISO 9000 or EN ISO/IEC 17025. In 2002, considering the new LTP, a new version of these prescriptions was prepared which came into force at the beginning of 2006. The goal of these guidelines is to set a framework for all RBTS which obliged the RBTS to put in place and maintain a quality management system allowing the preparation of blood components consistent with the quality requirements. The guidelines ensure the standardisation of the methods according to the actual state of the art and competency testing. These guidelines are shaped according to the process approach defined by the ISO 9001:2000 standard with 4 groups of processes,:

- management responsibility,

- resource management,

- product realization,

- measurement, analysis and improvement,

and describe their mutual interactions. As general standard, it covers the widest range of possible activities in a RBTS which must be supervised and analysed.

The content is a collection of guidelines, procedures and recommendations which determine how to perform the following activities:

- donor selection and blood donation,

- management of facilities, material and equipments,

- blood group serology and screening for infectious markers,

- preparation of blood components,

- quality control,

- release,

transport and distribution,

vigilance system.

The references used are the legal requirements (including [1] and [2]) and, specifically applied to laboratory activities, the ISO/IEC 17025:1999 and ISO 15189:2003 standards. They are mandatory for all RBTS and are regularly revised and updated if necessary by expert working groups of the RBTS and Swissmedic under the responsibility of the executive board.
They contain also criteria for medical assessment of blood donors, algorithms for HCV, HBV, HIV and syphilis screening, specifications of each blood component (used as reference by Swissmedic during their inspections) and different lists of admitted material and reagents for serological testing.

Currently, all blood components are leucocyte-depleted, and $90 \%$ of platelet concentrates (PC) are obtained by apheresis. Plasma for transfusion can only be released as fresh frozen plasma (FFP) after 4 months of storage in quarantine or after solvent/detergent treatment.

After each donation, the following tests are performed: antiHIV and HIV NAT, anti-HCV and HCV NAT, HBsAg, antiTreponema pallidum, alaninaminotransferase (ALAT) and blood grouping (ABO/Rhesus D).

The BTS SRC has also published recommendations for clinicians on autotransfusion and immunohaematology.

\section{Accreditation and/or Certification}

Most of the RBTS have now received an accreditation and/or a certification.

The accreditation which is based on the ISO/IEC 17025 standard includes the laboratory activities and covers also blood donation in several RBTS. It is carried out by the Schweizerische Akkreditierungsstelle (SAS). Control audits take place yearly, and after 5 years the accreditation must be renewed. About one third of the RBTS are additionally certified according to ISO9001:2000 standard by private, accredited organisations.

\section{Corrective and Preventive Actions / Risk Management}

According to the standards in place, each RBTS must perform corrective and preventive actions.

Collected data referring to donors, donations, vigilance and look-back procedures, post-donation information, quality controls, internal and external audit reports, complaints and failure management are regularly analysed. Management reviews assess the effectiveness of the quality management system, and corrective measures are performed, if necessary.

Reporting to Swissmedic is mandatory in case of unexpected events and quality deficiencies (for details, see below 'Haemovigilance' in 'Quality-Related Specifics').

The following preventive measures being implemented as precautionary measures, and coping with risk situations have been put in place in the last few years. They are mandatory for the BTS SRC and have been discussed with Swissmedic before implementation:

- universal leucocyte depletion (1999),

- exclusion of individuals who stayed longer than 6 months in the UK (2002),

- exclusion of transfused donors (2004). 
Table 1. National haemovigilance report 2002-2003

\begin{tabular}{lll}
\hline Blood components transfused & $\begin{array}{l}\text { Serious events } \\
\text { resulting in death }\end{array}$ & Life-threatening serious events \\
\hline RCC & 3 TACO & 1 IBPT, 1HTR, 1 Ana, 1 Hypo, 3 TACO \\
PC & 1 Bact.C. & 2 Ana, 1 PTP \\
FFP & - & 5 Ana, 2 TRALI \\
Diverse blood components & - & 1 Ana, 1 Hypo \\
\hline
\end{tabular}

Ana = Anaphylactic reactions; Bact.C $=$ bacterial contamination; HTR = haemolytic transfusion reaction; Hypo $=$ hypotensive transfusion reaction; $\mathrm{IBPT}=\mathrm{ABO}$-incompatible transfusions as a result of errors; $\mathrm{PTP}=$ post-transfusion purpura; $\mathrm{TACO}=$ transfusion-associated circulatory overload.

\section{National Reference Activities and Statistics}

The BTS SRC mandates a reporting office for monitoring mandatory look-back related to donors (seroconversion by regular donors) and related to patients (seroconversion after transfusion). The results must be transmitted to the health authorities. This office prepares a yearly report.

The BTS SRC mandates national reference laboratories in the following fields:

- immunohaematology for clarification of immunohaematological problems, determination of rare blood antigens and constitution of a list of donors with rare antigen constellations;

- infectious disease laboratory to confirm 'not negative' screening tests in donors;

- external quality control program for proficiency testing in immunohaematology and infectious disease serology (mandatory participation for all RBTS).

The RBTS compile data on collection, donors, epidemiology and finance reporting for submission to the BTS SRC.

\section{Quality-Related Specifics}

\section{Cost Containment and Cost Control}

Blood products are considered as drugs by law and therefore have a fixed production price (for sale by RBTS to hospitals) and a fixed public price (sale by hospitals to patients). These prices are based on the real costs for recruitment, blood collection, production, testing, manpower, materials and administrative work. However, research activities are not included. Currently only two main products (red cell concentrates (RCC) and FFP) are regulated. Regulations for PC will follow within the next few months.

In comparison with other European countries, the costs for a $\mathrm{RCC}$ are in the lower while the price for FFP is in the upper range. The price of plasma for fractionation, sold at a world market price, has to be negotiated each year with three different private plasma fractionation companies. There is no national self-sufficiency program for these products.
The BTS SRC must check regularly that the system is cost-efficient.

The price for pre-transfusion controls and cross-matching is fixed by the government and paid directly by the patients.

Health insurance is mandatory for each person living in Switzerland and covers all expenses mentioned above.

\section{Haemovigilance}

The LTP enacted in 2002 confers the responsibility for the national haemovigilance system to Swissmedic task of which is to assure and improve safety, quality and efficacy of haemotherapy and achieve better patient care. The haemovigilance system covers all aspects of transfusion medicine - starting with donor selection criteria and ending with the evaluation of the clinical effects of transfusion. Every suspected transfusion reaction, primarily

- transfusion-transmitted infections such as viral, parasitical and bacterial infections,

- immune complications, comprising haemolytic reactions (acute (aHTR) and delayed haemolytic transfusion reaction (dHTR)), transfusion-related acute lung insufficiency (TRALI), febrile, allergic and anaphylactic reactions,

- cardiovascular and metabolic complications such as transfusion-associated circulatory overload (TACO), hypothermia, hypocalcaemia and hyperkalaemia,

- errors and near-misses as well as

- a missing clinical effect

must be reported by hospitals. Blood centres must report all quality problems, e.g.:

- safety risks for blood donors, i.e. all events which could jeopardize donor health,

- all mix-ups involving donors or donations,

- erroneously released products and wrongly labelled products,

- release of blood components which do not fulfil specification requirements,

- defective materials and reagents, faulty testing.

The BTC operate under a Swissmedic licence. Therefore, Swissmedic is also responsible for taking actions if serious incidents occur. The cantons are responsible for taking legal ac- 
tion in regard to malpractice aspects occurring in hospitals, i.e. mistransfusions. This allows Swissmedic to operate the haemovigilance system with a 'no blame' culture with regard to medical practice aspects. The 'no blame' culture is one of the pillars of the haemovigilance system.

The law requires every institution with activities in transfusion medicine to appoint a responsible person for haemovigilance and to implement a quality system. This person is the contact person for Swissmedic and is responsible to ensure reporting to Swissmedic. The attending physician should report any suspected transfusion reaction to the haemovigilance designate in his institution. After the clinical and laboratory assessments are complete, the Swissmedic haemovigilance reporting form is forwarded either directly to Swissmedic or the supplying BTC, which forwards the report to Swissmedic. The report includes full particulars of the clinical situation and results of laboratory investigations. Reports of high quality are a prerequisite for proper assessment and deductions resulting in evidence-based recommendations. The two reporting paths, one directly to Swissmedic and one involving the supplying BTC co-exist because some BTC are actively involved in cross-matching blood components for patients. Important is that supplying BTC are immediately informed whenever the quality of the blood component is queried, e.g. if a bacterial contamination is suspected, thus enabling them to quarantine other potentially affected blood components. Time frames for reporting are defined by law. Serious events must be reported as soon as possible, at least within 15 days, all other events within 60 days.

Swissmedic has published the first national haemovigilance report in 2002-2003 (table 1); the 2004 report is on the way. The results are comparable to other haemovigilance schemes. Serious adverse events are due to anaphylactic reactions, TACO, TRALI, ABO-incompatible transfusions as a result of errors and bacterial contamination.

The first experience with the haemovigilance systems highlights the importance of proper evaluation after transfusion complications, the need for implementation of optimal use of blood components guidelines and the necessity of a quality system for blood administration with stringent rules for patient identification. Another important issue is open communication among all players involved in transfusion medicine.

\section{Contingency Plans}

An emergency planning for all imaginable crises such as a pandemic situation, a major accident, a terrorist attack or a natural disaster is established in coordination with the authorities, the rescue organisations and in a combined board of army and civil organisations.

The BTS SRC has a permanent committee (director, medical director and three RBTS directors) with dedicated staff for all those situations, which is entitled to manage immediately all RBTS activities in emergency cases. The main task is to guar- antee the communication with the governmental emergency boards, with the hospitals (need for blood supply), with the RBTS and with the public in order to get the right products in the right amount at the right place and time. The main focus is to have enough blood products but not to create an excess at short notice and a shortage 3 months later.

Special agreements with the government compel the main suppliers to warrant a minimal stock of blood bags for an extra period of 2 months.

\section{Specific Topics}

\section{Management of Immunological Risks and Prevention}

\section{Background}

Immunological risks of transfusion (at least) include immunisation against allogeneic antigens, aHTR and dHTR, febrile non-haemolytic transfusion reactions (FNHTR), allergic reactions, TRALI, post-transfusion purpura (PTP) and transfusion-associated graft versus host disease (TA-GVHD). These diverse risks of transfusion often are not sufficiently elucidated at the pathophysiological level. This means that there are often insufficient data for the development of evidence-based management or prevention. The following paragraph again confines to Swiss peculiarities.

\section{Management of Immunological Risks}

Apart from the prescriptions of the BTS SRC and the recently established national haemovigilance system, there are still no specific guidelines implemented in Switzerland, which countrywide may cover the workup, treatment and prevention of a transfusion reaction. However, in recent years and particularly responding to the LTP enacted in 2002, major hospitals re-activated or set up transfusion committees which updated or started developing in-house guidelines, and the two national expert societies (BTS SRC and Swiss Association of Transfusion Medicine (SATM)) intensified their co-operation in this field.

The attending physician is responsible for workup and treatment of any adverse transfusion reaction. The requirements given by the national haemovigilance system and, if applicable, in-house guidelines have to be considered.

Finally, a national rare donor program for RCC (http://rare. bsd-be.ch; password available on demand) and a national registry of HLA- and HPA-typed platelet apheresis donors have been established on request of the BTS SRC.

\section{Prevention of Immunological Risks}

At present, there are no specific national guidelines available. However, the BTS SRC has published recommendations for pre-transfusion tests to be performed in the RBTS. The recommendations are in accordance with GMP and good laboratory practice (GLP) guidelines and include specifications of 
serological blood group testing as well as some advice concerning blood transfusion and quality management. However, guidelines for the actual process of transfusion (who, when, what, how) have to be established by the hospitals.

Immunisation against Allogeneic Antigens

In addition to the standard of ABO- and Rhesus D-identical transfusions, it is recommended to avoid a Rhesus C, c, E, e and Kell immunisation of female children and women of childbearing age by using compatible transfusions of cellular blood products. For patients who are expected to be transfusion-dependent for a longer period of time and in agreement with their attending physicians, it is recommended to transfuse RCC being compatible for the 'most important antigens'. In case of transfusion of Rhesus D-positive PC to Rhesus-negative recipients, an adapted Rhesus immunoprophylaxis has to be considered.

\section{aHTR and dHTR}

The prevention of aHTR and dHTR is based on proper identification (as a rule 'second set of eyes') of the transfusion recipient and the pre-transfusion blood specimens being used for serological compatibility tests.

In 2002 the SATM published a statement concerning the proper identification of the patient and his designated blood units at the bedside [3]. The SATM concluded that there are different possible approaches, including an improved training of the staff; more frequent and stringent controls at the bedside; consistent implementation of a serologic bedside test or introduction of electronic devices, to verify and document the identity of patients and blood products. Every hospital has to determine an approach which is locally most appropriate and realisable. Therefore, in Switzerland a bedside test is not mandatory. Pre-transfusion testing at least includes the type and screen (T and $\mathrm{S}$ ) procedure or the serologic cross-matching including the antiglobulin test. In this context the main Swiss particularities with respect to the published recommendations by the BTS SRC (being often adopted by hospitals) are as follows: It is not accepted to perform pre-transfusion tests using ambiguously labelled blood specimens; it is recommended to perform blood group testing of the patient using two blood specimens being drawn on separate occasions; for antibody screening and identification the red cell panels used have to be positive for the following antigens: D, C, Cw, c, E, e, M, N, S, s, P1, K, k, Kp(a), Le(a), Le(b), Fy(a), Fy(b), Jk(a), Jk(b) and preferably $\mathrm{Lu}(\mathrm{a})$; at least one of them has to be homozygous for the antigens D, C, c, E, e, S, s, Fy(a), Fy(b), Jk(a) and Jk(b); commercial panels have to be negative for the rare antigens $\mathrm{Mg}$, $\mathrm{Wr}(\mathrm{a})$ and $\mathrm{Vw}$; if clinically significant antibodies are excluded by means of $\mathrm{T}$ and $\mathrm{S}$ and if there is no record of previous detection of such antibodies, at least ABO- and Rhesus D-compatible red cell units may be released; otherwise the antibody has to be identified, and cross-matching of antigen-negative units is prescribed.

\section{FNHTR}

In Switzerland pre-storage leucocyte depletion (residual WBC $<1 \times 10^{6}$ per unit) is mandatory since July 1999 . Although it has primarily been implemented in the context of preventive measures against a possible transmission of variant Creutzfeld-Jacob disease (vCJD), it has also been shown to be preventive for FNHTR.

\section{Allergic Reactions, TRALI, PTP, TA-GVHD}

A task force of the BTS SRC is currently developing a strategy for prevention of immune TRALI. There are no Swiss peculiarities in workup, treatment and prevention of allergic reactions, PTP and TA-GVHD.

\section{Strategies for Prevention of Transmission of Viruses, Bacteria and Parasites / NAT}

Measures for preventing transfusion-transmitted diseases are implemented in all stages of the whole process ranging from the donor selection to the transfusion.

\section{Donors}

The high safety of the blood products begins with a careful selection of blood donors. In this context, the proportion of firsttime to repeat donors is important. During the last 10 years the donor population consisted primarily of voluntary nonremunerated repeat donors, with only $5 \%$ being first-time donors.

Other important points to gain safe donors are the medical questionnaire and examination of the blood donor. Today 21 questions are listed in the questionnaire, dealing with different issues such as donor's risk situations, travelling in foreign countries and possible diseases or infections in the past or present. In addition, each donor gets information sheets on the same matter.

In 2002, the health authorities decided to defer blood donors who had stayed longer than 6 months in the UK after 1980, and in 2004, those with a history of previous blood transfusion after 1980. Theses measures were implemented in order to deal with the potential risk of transmission of prion infections such as vCJD.

\section{Donation}

Strict hygiene conditions are essential to prevent potential contaminations during the donation process. Standardised disinfection procedures of the venipuncture site are in place and must be carefully followed. Furthermore, pre-donation sampling is mandatory since April 2004.

\section{Production of Blood Components}

The strict hygiene measures implemented during donation must also be adhered to during the blood component production process and the handling of blood components. 
In July 1999, universal leucocyte depletion was implemented. Although the crucial reason was to reduce the risk of prion transmission, this procedure was already known to reduce significantly the rate of transfusion-transmitted infections by white cell-bound viruses such as CMV, EBV, HTLV I/II and HHV 8.

\section{Screening Tests}

Each blood donation must be screened for possible contamination with blood-borne viruses. Currently, the following serological screening tests are mandatory by law: anti-HIV $1 / 2$, anti-HCV, HBsAg, anti-Treponema pallidum and ALAT. The upper threshold for the ALAT is $80 \mathrm{IU} / \mathrm{ml}$ and $100 \mathrm{IU} / \mathrm{ml}$ for women and men respectively. These screening tests are performed locally by 9 out of 13 RBTS, whereas the 4 remaining RBTS have outsourced their laboratory tests to neighbour hospital laboratories or other RBTS.

\section{Confirmation Tests}

If a sample reveals a reactive result in a screening test at least twice, it must be confirmed by the national reference laboratory. The confirmation algorithms for the 3 blood-borne viruses HBV, HCV and HIV are as follows:

- Samples reactive for HBsAg are analysed with a different HBsAg enzyme immunoassay and, if further reactive, tested by a neutralization assay. In addition, anti-HBc testing is performed.

- Samples reactive for anti-HCV are confirmed with a different anti-HCV enzyme immunoassay and with a HCV recombinant immunoblot assay.

- Samples reactive for HIV are confirmed with a different anti-HIV 1/2 test, a p24 Ag assay and a HIV blot assay.

\section{$N A T$}

In July 1999, HCV NAT became mandatory. Approximately 3 years later HIV 1-NAT was introduced.

NAT for HCV and HIV-1 are currently performed by 7 independent laboratories using mini-pools including 16-49 donations. These centres test between 12,000 and 220,000 donations per year. All tests are currently performed with the HCV and HIV-1 Cobas Ampliscreen assays (Roche Diagnostics, Rotkreuz, Switzerland).

According to the requirements of the health authorities, the minimal analytical sensitivities for HCV and HIV in a single donation are 5,000 and 10,000 IU/ml respectively.

The extraction method of nucleic acid prior to NAT is not determined by the authorities. Therefore, several methods are in use ranging from the hand Roche method over the single Qiagen silica columns to fully automated Qiagen plates and pipetting robots. However, each method must be validated locally with the guidelines of the German Paul Ehrlich Institute to demonstrate that the requested sensitivities in mini-pools are achieved.

\section{Incidence / Residual Risk}

Incidence and residual risks have been calculated according to the model of Schreiber [4].

From 1996 to 2003 the incidence rate for HCV decreased continuously, whereas no significant decrease in the incidence rate of HIV and HBV has been observed.

The residual risk has been calculated with NAT window periods for HCV between 1999 and 2003 and for HIV between 2002 and 2003. For the period of 2002-2004, it was estimated to be 1 in 2,300,000 for HIV, 1 in 2,200,0000 for HCV and 1 in 141,000 for HBV [5].

Since the introduction of the HCV NAT in 1999 (with approximately 3.5 million donations tested) 2 window period cases, both from repeat donors, have been detected, but for HIV no case has yet been found (approximately 1.4 million donations tested). These data correlate well with data from several other European countries and the USA [6]. According to international studies, the estimated residual risk for HBV between 1996 and 2003 is significantly higher than for HCV and HIV. In Switzerland, HBV is the virus with the highest risk for transmission to blood recipients. A current study by the RBTS Berne and the RBTS Vaud should help to elucidate which additional test (anti-HBc and/or HBV NAT in mini-pool) should be preferred to improve blood safety.

\section{Future Challenges}

The main challenges for the BTS SRC (and the Swiss transfusion medicine community) in the next years are:

- Maintaining the blood supply all over the country (self-sufficiency). The demographic situation shows that the average age of the regular blood donor population is growing continuously. This could lead to a shortage of donors if we are not able to motivate the young generations.

- Medical personnel and laboratory staff. In Switzerland no specific formation for transfusion specialists exists; specific transfusion activities are taught during a maximum of 1 year during the formation in haematology. Furthermore, young doctors often prefer remaining in a hospital because the specific job in a RBTS is not considered as attractive enough without contact to patients. We should be able to offer positions combining supervision of donation, production and laboratory testing and clinical activities and/or participation to research projects. Apart from that, we must improve special training in transfusion medicine.

- Financial aspects. Health costs are rising fast in Switzerland as in most countries. Therefore, we will have to be even more efficient despite possible future implementations of new and expensive technologies dealing with production, testing and pathogen inactivation of blood components. Further concentration and cooperation between the transfusion actors are needed. 


\section{References}

1 Guide to the Preparation, Use and Quality Assurance of Blood Components. Strasbourg, Council of Europe Publishing, 2006.

2 PIC/S GMP Guide for Blood Establishments. Pharmaceutical Inspection Convention. 2004; PE 005-2. www.picscheme.org/indexnoflash.php? $p=$ guides\#.

3 Bedside-Tests bei allen Erythrozyten-haltigen Bluttransfusionen. Stellungnahme der Schweizerischen Vereinigung für Transfusionsmedizin (SVTM) Schweizerische Ärztezeitung 2002;83:273-274.
Schreiber GB, Busch MP, Kleinman SH, Korelitz JJ: The risk of transfusion-transmitted viral infections. The Retrovirus Epidemiology Donor Study. N Engl J Med 1996;334:1685-1690.

5 Niederhauser C, Schneider P, Fopp M, Ruefer A,

Lévy G: Incidence of viral markers and evaluation of the estimated risk in the Swiss blood donor population from 1996 to 2003. Eurosurveillance 2005; $10: 7-8$.
6 Coste J, Reesink HW, Engelfriet CP, Laperche S, Brown S, Busch MP, Cuijpers HT, Elgin R, Ekermo B, Epstein JS, Flesland O, Heier HE, Henn G, Her nandez JM, Hewlett IK, Hyland C, Keller AJ, Krusius T, Levicnik-Stezina S, Levy G, Lin CK, Margaritis AR, Muylle L, Niederhauser C, Pastila S, Pillonel J, Pineau J, van der Poel CL, Politis C, Roth WK, Sauleda S, Seed CR, Sondag-Thull D, Strame SL, Strong M, Vamvakas EC, Velati C, Vesga MA, Zanetti A: International forum: 1. Vox Sang 2005; 88:289-298. 